\title{
THE
}

\section{Three-dimensional propagation effects near the mid-Atlantic Bight shelf break (L)}

\author{
Kevin B. Smith \\ Chris W. Miller \\ Anthony F. D'Agostino \\ Brian Sperry \\ James H. Miller \\ University of Rhode Island, miller@uri.edu
}

See next page for additional authors

Follow this and additional works at: https://digitalcommons.uri.edu/gsofacpubs

Terms of Use

All rights reserved under copyright.

\section{Citation/Publisher Attribution}

Smith, K. B., Miller, C. W., D'Agostino, A. F., Sperry, B., Miller, J. H., \& Potty, G. R. (2002). Three-dimensional propagation effects near the mid-Atlantic Bight shelf break (L). Journal of the Acoustical Society of America, 112(2), 373-376. doi: 10.1121/1.1490559

Available at: https://doi.org/10.1121/1.1490559

This Article is brought to you for free and open access by the Graduate School of Oceanography at DigitalCommons@URI. It has been accepted for inclusion in Graduate School of Oceanography Faculty Publications by an authorized administrator of DigitalCommons@URI. For more information, please contact digitalcommons-group@uri.edu. 


\section{Authors}

Kevin B. Smith, Chris W. Miller, Anthony F. D'Agostino, Brian Sperry, James H. Miller, and Gopu R. Potty 


\section{Three-dimensional propagation effects near the mid-Atlantic Bight shelf break (L)}

Kevin B. Smith, Chris W. Miller, Anthony F. D’Agostino, Brian Sperry, James H. Miller, and Gopu R. Potty

Citation: The Journal of the Acoustical Society of America 112, 373 (2002); doi: 10.1121/1.1490559

View online: https://doi.org/10.1121/1.1490559

View Table of Contents: http://asa.scitation.org/toc/jas/112/2

Published by the Acoustical Society of America

\section{Articles you may be interested in}

Effect of shelf break front on acoustic propagation

The Journal of the Acoustical Society of America 119, 3346 (2006); 10.1121/1.4786456

An analysis of the three-dimensional sound field in a penetrable wedge with a stratified fluid or elastic basement The Journal of the Acoustical Society of America 93, 1319 (1993); 10.1121/1.405417 


\title{
Three-dimensional propagation effects near the mid-Atlantic Bight shelf break (L)
}

\author{
Kevin B. Smith, Chris W. Miller, and Anthony F. D'Agostino \\ Naval Postgraduate School, Monterey, California 93943 \\ Brian Sperry \\ Woods Hole Oceanographic Institute, Woods Hole, Massachusetts 02543 \\ James H. Miller and Gopu R. Potty \\ University of Rhode Island, Narragansett, Rhode Island 02882
}

(Received 14 February 2002; accepted for publication 7 May 2002)

\begin{abstract}
Significant three-dimensional (3-D) environmental variability exists in the vicinity of the shelf break along the mid-Atlantic Bight. This study examines the influence of azimuthal coupling due to this variability on acoustic propagation in this region. Numerical studies employing a 3-D ray code, a hybrid ray-mode code, and a 3-D parabolic equation model are used to study the significance of azimuthal coupling on various propagation paths. These paths include up-slope, slant-slope, and cross-slope propagation. The numerical analysis suggests that, for the propagation ranges less than $60 \mathrm{~km}$ examined, the influence of azimuthal coupling is negligible compared to the inherent uncertainty in the environment itself. (C) 2002 Acoustical Society of America.
\end{abstract}

[DOI: $10.1121 / 1.1490559]$

PACS numbers: 43.30.Dr, 43.30.Re, 43.30.Zk [SAC-B]

\section{INTRODUCTION}

Continental slopes represent some of the steepest largescale bathymetric features in the world's oceans, averaging 2-3 degrees and extending for several miles out from the continental shelf. The boundary between these regions and the shallow, littoral zones on the shelf is the shelf break, typically an area of significant oceanographic variability. Such three-dimensional environmental variability exists in the vicinity of the shelf break along the mid-Atlantic Bight and is characterized by relatively strong bathymetric slopes, cold shelf water interacting with warm Gulf Stream waters, and diurnal generation of internal solitary waves onto the shelf. The influence of such a highly variable environment on acoustic propagation is of great interest as is the impact on inversion techniques such as tomography and localization, and sonar system performance estimations.

In the summer of 1996, the Office of Naval Research sponsored an acoustic field study, called the Shelf Break Primer, off the coast of New Jersey near the mid-Atlantic Bight. Details of the experimental design and data collection are provided by Lynch et al. (1997).

This letter provides an overview of several different numerical propagation studies performed for this region. Specifically, each study examined the predicted influence of azimuthal coupling due to the three-dimensional character of the environment on acoustic propagation. A 3-D ray code was used to estimate horizontal refraction of propagation paths and differences in arrival times when contrasted with simple 2-D ray calculations. A hybrid ray-mode code was also used to investigate the influence of the refracted paths on mode amplitude and phase. The modes were assumed to be adiabatic in this treatment. Finally, a fully coupled 3-D parabolic equation model was used to investigate travel time differences and to determine the dominant mechanism for 3-D effects.

\section{ENVIRONMENTAL/EXPERIMENTAL DESCRIPTION}

The primary assets used in the experiment consisted of three tomography arrays with sources moored near the bottom. All of these sources were deployed on the slope about $30 \mathrm{~km}$ seaward of the shelf break at depths of $300-350 \mathrm{~m}$. On the shelf were deployed two, 16-element vertical line arrays (VLAs). The depth of the lowest array element was 3 $\mathrm{m}$ off the seafloor with $3.5-\mathrm{m}$ element separation for a total aperture of $52.5 \mathrm{~m}$. The configuration of the sources and VLAs relative to the local bathymetry based on standard USGS contours is displayed in Fig. 1.

In addition to the acoustics, several assets were continuously devoted to environmental assessment. These included a string of thermistors located near the southwest corner of the experiment and a nearly continuous towing of a highresolution SeaSoar (Gawarkiewicz et al., 2001). This device was able to measure temperature, salinity, and various other water properties as a function of depth. During the deployment, four cross-shelf transects, roughly $45 \mathrm{~km}$ in length, were conducted daily. Each transect was separated by $10 \mathrm{~km}$ in the along-shelf direction to resolve the three-dimensional structure of the shelf break region. An example sound speed profile generated from such data is displayed in Fig. 2. Note the cold "tongue" of shelf water extending out over the shelf break. Gradients as large as $2 \mathrm{~s}^{-1}$ can be seen at the boundaries of this protrusion.

\section{NUMERICAL RESULTS}

Several different source/receiver tracks were investigated in the analysis, including two directly upslope onto the shelf (SW2NW, SE2NE) and two crossing diagonally across the shelf (SW2NE, SE2NW). 


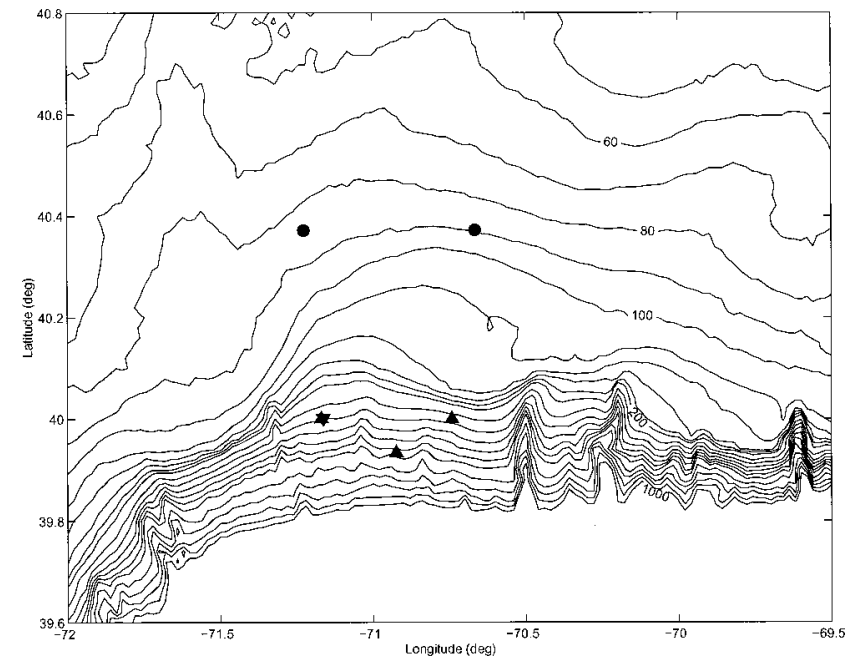

FIG. 1. Bathymetry and location of deployed acoustic elements for Primer experiment. The circles indicate the locations of the vertical line arrays (VLAs), the upright triangles indicate the locations of the 400-Hz tomography sources, and the upside-down triangle indicates the location of the $240-\mathrm{Hz}$ tomography source (co-located with the southwest $400-\mathrm{Hz}$ source).

The majority of the ray modeling work was done prior to the actual experiment in an attempt to provide some $a$ priori knowledge of the expected influence of 3-D propagation (D'Agostino, 1996). Therefore, the environmental characterization, specifically the sound speed profiles, was based on empirical knowledge of shelf break regions. The bottom was treated as a simple reflector. To account for the critical angle effects, no rays which interacted with the bottom at any point along the path with grazing angles greater than $\sim 25$ degrees were kept. This led to ray vertical launch angles between -7 and +7 degrees with a chosen resolution of 0.01 degree.

The 3-D ray code used was a modified version of HARPO adapted to compute the necessary properties of eigenrays (see D'Agostino, 1996). The eigenray pressure magnitudes, phases, and travel times, including the effects of the boundary reflections, ray turning, and raytube spreading, were then computed.

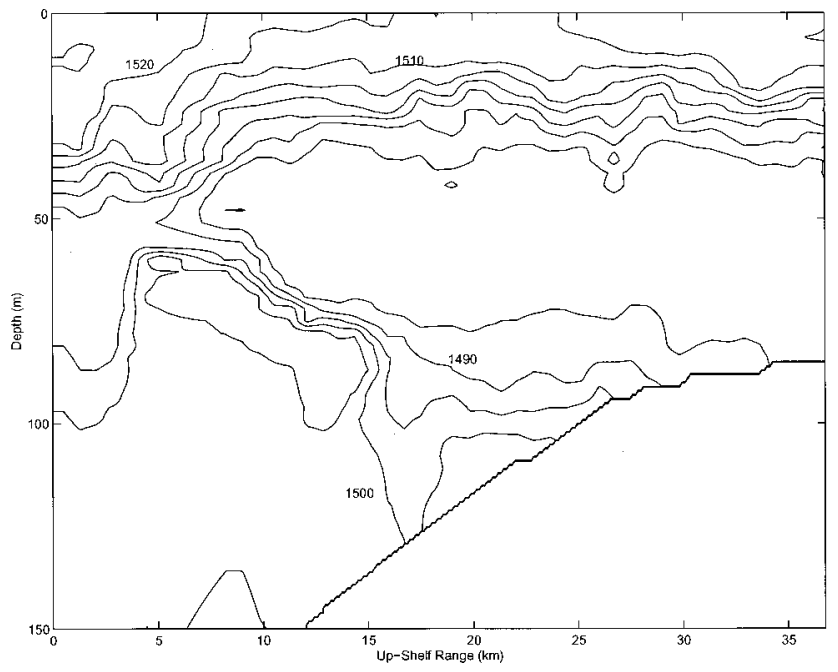

FIG. 2. Two-dimensional slice of sound speed profiles as computed from temperature and salinity measurements made by the SeaSoar.
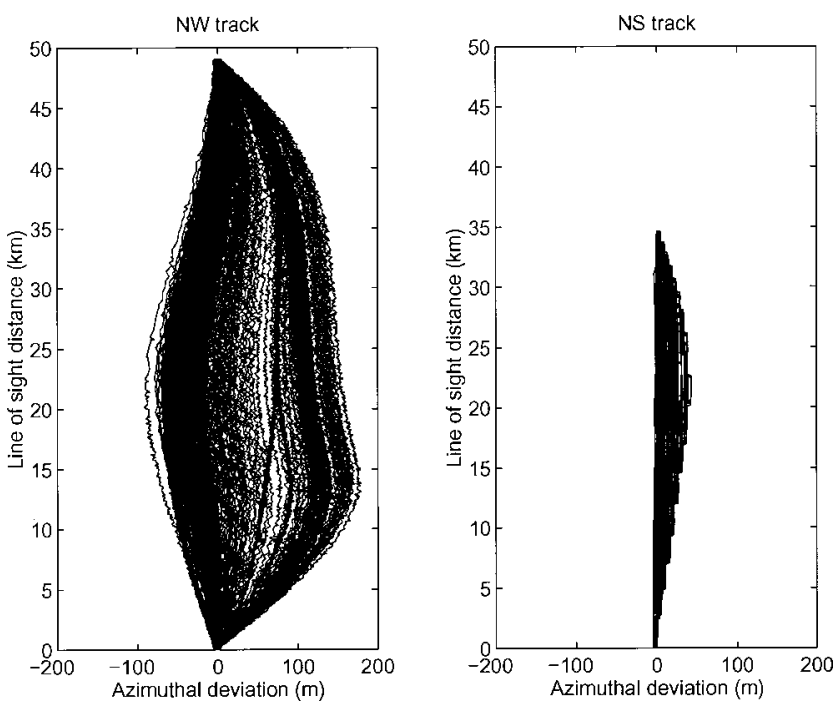

FIG. 3. Plan view of eigenray paths from the southeast source position to the northwest (left) and northeast (right) VLA positions.

Figure 3 is a plan view of the eigenray geometries showing the azimuthal deviations from the line-of-sight as a function of range for all the 3-D eigenrays for both the SE2NE and SE2NW cases. The SE2NE track bisects the ocean front and bathymetry at approximately right angles and thus the azimuthal deviations are minimal, less than $50 \mathrm{~m}$ over $\sim 30$ $\mathrm{km}$. The SE2NW track bisects the ocean front and bathymetry along a diagonal and is more strongly affected by 3-D effects from both the ocean frontal structure and the bathymetry. Upon inspection, it was found that the horizontal refraction of the steep eigenrays is controlled by the bathymetry while the small-angle eigenrays were more affected by the frontal structure. Still, the maximum horizontal deviation is less than $150 \mathrm{~m}$ over $\sim 45 \mathrm{~km}$.

The ray-mode analysis was conducted after the experiment. Because bottom interactions were significant and affect both the ray-mode and PE calculations, it was important to provide a good characterization of the sea-floor acoustic properties. Cores have been taken at nearby sites on the shelf (Hathaway et al., 1976). Additional analysis of the ambient noise field and explosive SUS charges measured on the northeast array provide similar values for sediment sound speed (Potty et al., 2000).

Horizontal mode refraction calculations were made of the SE2NE and SW2NE paths. Note the bathymetry for the direct upslope tracks are identical to that used in the previous ray results. The SW2NE track has a similar inverted bathymetric influence as the previous SE2NW track. For each track, two different environments were considered. Both are based on the objectively analyzed SeaSoar data product, which is a 3-D block of temperature and salinity over the entire SeaSoar domain (Sperry, 1999). The first, a cross-shelf reference slice, was created by averaging all of the SeaSoar data in the along-shelf direction, as well as over all seven days of SeaSoar data collection. This gave a 2-D cross-shelf slice that ended up very closely resembling climatology and the historical database used in the ray work. This single slice was then replicated in the along-shelf direction to create the reference environment. The reference case then has relatively 

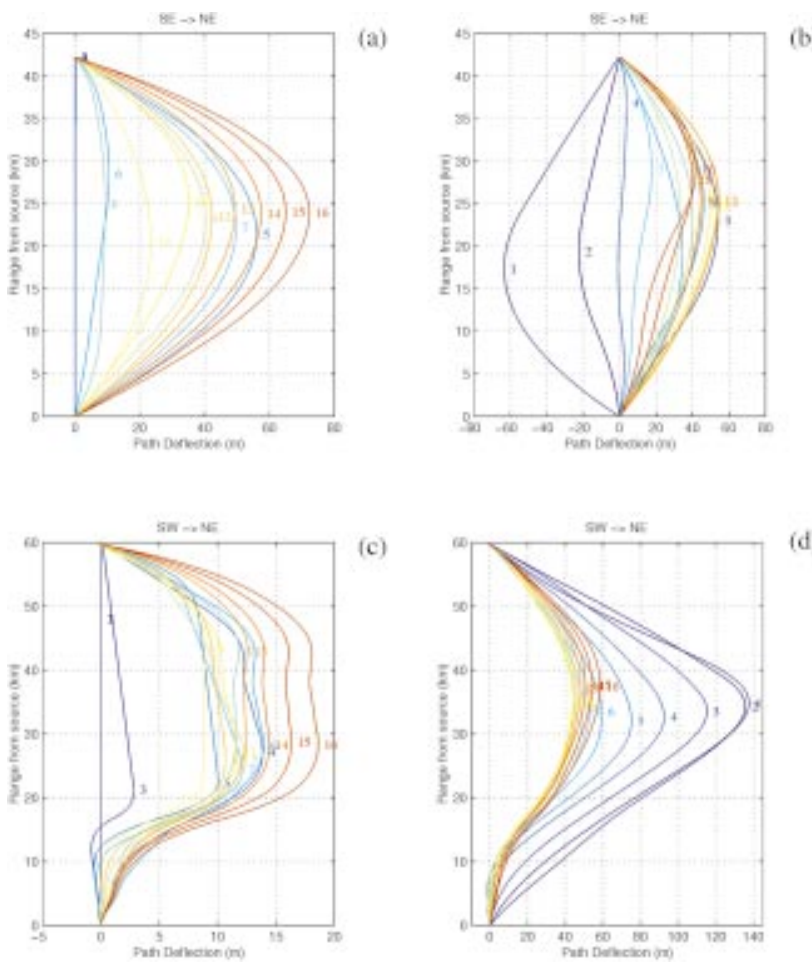

FIG. 4. Modal horizontal deflection paths: (a) SE2NE track for the reference profiles; (b) SE2NE track for the Day 7/31 environment; (c) SW2NE track for the reference profiles; and (d) SW2NE track for the Day 7/31 environment.

smooth variations in the cross-shelf direction and no variability in the along-shelf direction. The second environment, simulating Day $7 / 31$ of the experiment, was taken directly from the objectively analyzed SeaSoar data containing the full 3-D variability of the oceanography.

Figure 4 shows the resulting mode paths for the two environments and two source/receiver paths. For the reference propagation case [Figs. 4(a) and (c)], the lowest two to three modes make the trip with no refraction at all. There is no bottom interaction for these modes, and the cross-slope variability in phase speeds is slight. The more bottominteracting higher modes are all refracted to varying degrees toward the right, in accordance with local bathymetric gradients, consistent with the ray paths in Fig. 3. Although the deflections are as great as $70 \mathrm{~m}$ from the direct path, the total change in path length is no more than one meter in the worst case. Note also that this is similar in magnitude and effect to the SE2NW ray results.

To compute the influence of 3-D propagation using a full-wave model, the 3-D parabolic equation (PE) algorithm of Smith (1999) was used. Four different tracks were studied with this method: SW2NW, SE2NW, SW2NE, and a fourth, imaginary track from the southeast source directly west for $60 \mathrm{~km}$, hereafter referred to as the SE2W track, in an attempt to generate a stronger 3-D effect.

For travel time predictions, multiple frequencies were computed, specifically 32 frequencies over a $32-\mathrm{Hz}$ bandwidth centered at $400 \mathrm{~Hz}$. This bandwidth and frequency sampling were determined as minimal for travel time predictions in this region. Figure 5 displays the data from the reference environment along the SE2NW track after plane-
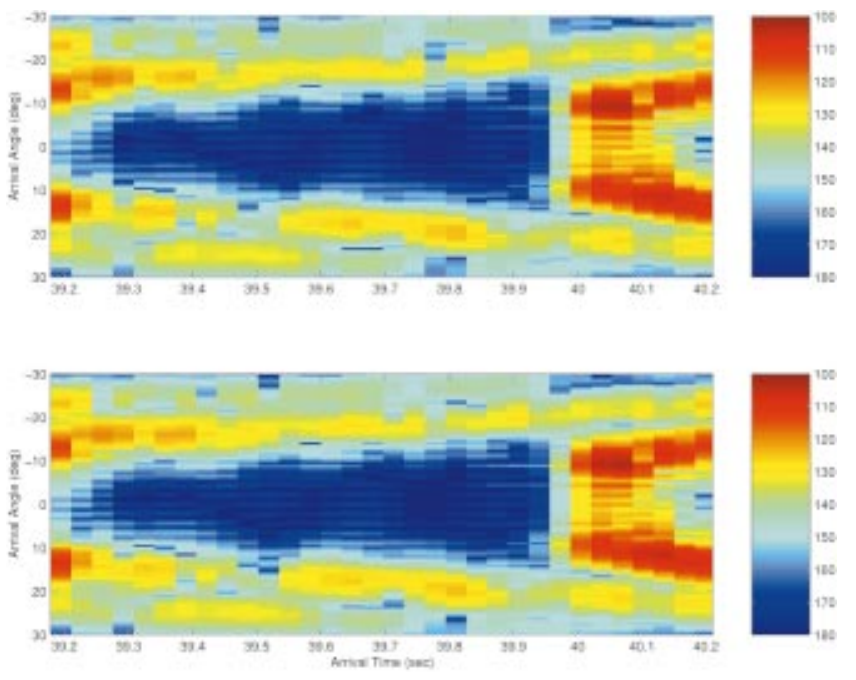

FIG. 5. A comparison of the arrival time structure versus arrival angle between the 2-D (upper plot) and 3-D (lower plot) PE models for the SE2NW track with the reference 3-D interpolated sound speed field.

wave beamforming in the vertical. Note the numerical wraparound effect due to insufficient frequency sampling, so that the first arrivals and loudest part of the signal appear at $\sim 40$ s. In this case, no significant differences exist with variations on the order of $\pm 5 \mathrm{~dB}$ in level and $< \pm 25 \mathrm{~ms}$ in travel times (less than the resolution of the calculation here). This is consistent with similar values found in the ray analysis.

\section{SUMMARY}

Both the 3-D ray and ray-mode solutions predicted negligible 3-D influences, due either to bathymetry or sound speed, in the directly upslope direction, SE2NE. Path deflections were typically $<60 \mathrm{~m}$ with travel time variations $<\frac{1}{2}$ ms. This is consistent with the lack of noticeable 3-D influences on the SW2NW results obtained from the PE model.

Both the 3-D ray code and the ray-mode calculations suggest horizontally deflected paths that may be separated from the direct line-of-sight path by as much as $100 \mathrm{~m}$ or more for diagonal cross-slope propagation. However, in each case this accounts for less than a 2-m change in total path length. Such path length variations contribute only as much as $\sim 1 \mathrm{~ms}$ difference in travel time over a $\sim 40-\mathrm{km}$ path. Such a difference would be on the order of a total sound speed perturbation of $\sim 0.1 \mathrm{~m} / \mathrm{s}$ or, similarly, a total temperature variation of $0.02{ }^{\circ} \mathrm{C}$. Thus, the environmental resolution required to clearly identify such phenomena is not currently achievable.

Both the ray and ray-mode analyses indicated that the strongest 3-D effect of the environment was due to bathymetry gradients near the shelf break. Consequently, the highest modes (and highest propagation angles) were deflected the most in a manner consistent with a wedgelike boundary. However, all the numerical results also indicate that the lowest modes contain the most travel time sensitivity and variability, even though their corresponding paths are more azimuthally stable. Considerably more variability and 3-D influences were observed in the ray-mode analysis when the more complex 3-D sound speed field was used instead of the 
symmetrical reference environment, as may be expected. Still, a general statement may be made that travel time fluctuations in this region are highest for the lower modes and dominated by variations in the sound speed field along the track rather than azimuthal path variations along the track itself.

All of the travel time results, using a 3-D ray, ray-mode, or 3-D PE model, produced similar results. Essentially, travel time differences were not distinguishable on the resolution scale available. The only exception found was the hypothetical SE2W path which considered propagation approximately parallel to the shelf for $60 \mathrm{~km}$. In this case, travel time differences of hundreds of ms were observed. Because such large travel time differences would require total sound speed perturbations of $\sim 5 \mathrm{~m} / \mathrm{s}$ ( or $1^{\circ} \mathrm{C}$ ), the $3-\mathrm{D}$ influences along this path may be distinguishable from environmental uncertainty.

From all of the numerical analysis conducted, there appears to be no clear signature of azimuthal coupling in the acoustic propagation. Horizontal arrival angles do provide some evidence of azimuthal refraction. However, for these environments and ranges, unrealistically long horizontal arrays would be needed to resolve such features. Furthermore, the maximum distances of propagation did not exceed any mode horizontal shadow zones and no other 3-D propagation phenomenon produces anything distinctive which differentiates it from a variable 2-D propagation path.

D'Agostino, A. (1996). "Simulation of 3-D acoustic arrival structure in the Middle Atlantic Bight and its effect on the inverse tomography solution," Master's thesis, Naval Postgraduate School, June 1996.

Gawarkiewicz, G., Bahr, F., Beardsley, R., and Brink, K. (2001). "Interaction of a slope eddy with the shelfbreak front in the Middle Atlantic Bight," J. Phys. Oceanogr. 31, 2783-2796.

Hathaway, J. C., Schlee, J. S., and Poag, C. W. (1976). "Preliminary Summary of the 1976 Atlantic Margin Coring Project of the U.S. Geological Survey," Open File Report, No. 76-844.

Lynch, J. F., Gawarkiewicz, G. G., Chiu, C.-S., Pickart, R., Miller, J. H., Smith, K. B., Robinson, A. R., Brink, K. H., Beardsley, R., Sperry, B., and Potty, G. (1997). "Shelfbreak PRIMER-An integrated acoustic and oceanographic field study in the Middle Atlantic Bight," in Proceedings of International Conference on Shallow Water Acoustics, Beijing, China, 21-25 April, pp. 205-212.

Potty, G. R., Miller, J. H., Lynch, J. F., and Smith, K. B. (2000). "Tomographic mapping of sediments in shallow water," J. Acoust. Soc. Am. 108, 973-986.

Smith, K. B. (1999). "A three-dimensional propagation algorithm using finite azimuthal aperture," J. Acoust. Soc. Am. 106, 3231-3239.

Sperry, B. (1999). "Analysis of Acoustic Propagation in the Region of the New England Continental Shelfbreak," Ph.D. dissertation, Massachusetts Institute of Technology/Woods Hole Oceanographic Institution, June 1999. 